\title{
Collinear Limits in QCD from MHV Rules
}

\author{
T. G. Birthwright, E. W. N. Glover, V. V. Khoze and P. Marquard. \\ Department of Physics, University of Durham, Durham DH1 3LE, U.K. \\ E-mail: T.G.Birthwright@durham.ac.uk, E.W.N.Glover@durham.ac.uk, \\ Valya.Khoze@durham.ac.uk, Peter.Marquard@durham.ac.uk
}

\begin{abstract}
We consider multi-parton collinear limits of QCD amplitudes at tree level. Using the MHV formalism we specify the underlying analytic structure of the resulting multi-collinear splitting functions. We derive general results for these splitting functions that are valid for specific numbers of negative helicity partons and an arbitrary number of positive helicity partons (or vice versa).
\end{abstract}

KEYWORDS: QCD, Supersymmetry and duality, Hadronic colliders. 


\section{Introduction}

The 'MHV rules' approach proposed in Ref. [1], has led to the establishment of a new and powerful framework for computing large classes of previously unknown treelevel and one-loop scattering amplitudes in gauge theories, in a compact form, and without appealing to Feynman diagrams.

In this paper, we apply the MHV rules to study the singular limits of QCD amplitudes when $n$ partons (gluons and massless quarks) are simultaneously collinear. This continues the program started in our earlier work [2] where MHV rules were used to derive multi-collinear limits of amplitudes involving only gluons. Understanding the infrared singular behaviour of tree-level QCD amplitudes is a prerequisite for computing infrared-finite cross sections at fixed order in perturbation theory. In general, when one or more final state particles are either soft or collinear, the amplitudes factorise. The first factor in this product is a scattering amplitude that depends only on the remaining hard partons in the process (including any hard partons constructed from an ensemble of unresolved partons). The second factor is the splitting amplitude, it contains all of the singularities due to the unresolved particles. One of the best known examples of this type of factorisation is the limit of tree amplitudes when two particles are collinear. This factorisation is universal and can be generalised to more particles [3-7] and any number of loops [8].

One of the main points of our approach [2] is that, in order to derive all required splitting functions we do not need to know the full amplitude. Out of the complete set of MHV-diagrams contributing to the full amplitude, only a subset will contribute in the multi-collinear limit. This subset includes only those MHV-diagrams where all of the internal propagators go on-shell in the multi-collinear limit. Moreover, the functions multiplying these singular propagators in the splitting amplitude are constrained by the MHV rules to take a purely holomorphic form: they are functions which depend only on the holomorphic spinor products, $\langle i j\rangle$, of the right-handed (undotted) spinors and not on the anti-holomorphic ones $[i j]$. This points towards a simple twistor space picture for the multi-collinear limits, in terms of a degree-one curve in twistor space. The MHV rules approach also enables us to calculate infinite sequences of splitting amplitudes - with fixed numbers of negative helicity partons and arbitrary numbers of positive helicity ones, or vice versa.

The basic building blocks of the MHV rules approach [1] are the colour-ordered $n$-point vertices which are connected by scalar propagators. These MHV vertices are off-shell continuations of the maximally helicity-violating (MHV) $n$-gluon scattering amplitudes of Parke and Taylor $[9,10]$. They contain precisely two negative helicity gluons. Written in terms of spinor inner products [11], they are composed entirely of the holomorphic products $\langle i j\rangle$, rather than their anti-holomorphic partners $[i j]$,

$$
A_{n}\left(1^{+}, \ldots, p^{-}, \ldots, q^{-}, \ldots, n^{+}\right)=\frac{\langle p q\rangle^{4}}{\langle 12\rangle\langle 23\rangle \cdots\langle n-1, n\rangle\langle n 1\rangle},
$$


where we introduce the common notation $\left\langle p_{i} p_{j}\right\rangle=\langle i j\rangle$ and $\left[p_{i} p_{j}\right]=[i j]$. By connecting MHV vertices, amplitudes involving more negative helicity gluons can be built up.

The MHV rules for gluons [1] have been extended to amplitudes with fermions [12]. New compact results for tree-level gauge-theory results for non-MHV amplitudes involving arbitrary numbers of gluons [13-15], and fermions [12, 16, 17] have been derived. They have been applied to processes involving external Higgs bosons [18, 19] and electroweak bosons [20]. MHV rules have also been shown to work at one-loop level for supersymmetric theories [24]. Building on the earlier work of Bern, Dixon, Dunbar and Kosower [21, 22], there has been a remarkable progress in computing cut-constructible multi-leg loop amplitudes in $\mathcal{N}=4[23-29]$ and $\mathcal{N}=1[30-34]$ supersymmetric gauge theories. Encouraging progress has also been made using MHV rules for non-supersymmetric loop amplitudes [35, 36].

Remarkably, the expressions obtained for the infrared singular parts of $\mathcal{N}=4$ one-loop amplitudes (which are known to be proportional to tree-level results) were found to produce even more compact expressions for gluonic tree amplitudes [29, 37]. This observation led to the BCF recursion relations [38, 39] of Britto, Cachazo, Feng and Witten as well as extremely compact six-parton amplitudes [38, 40, 41]. These tree-level BCF recursion relations for massless particles have recently been generalised in two ways. In Refs. [42, 43] a new version of recursion relations was adopted to calculate all finite one-loop amplitudes in non-supersymmetric QCD. At the same time, Ref. [44] generalised BCF recursion relations to include massive particles at tree level.

A comprehensive list of references and a more detailed discussion of recent developments can be found in the recent review [45]. This progress has been stimulated by the original proposal of Witten in [46] of a weak-to-weak coupling duality between a perturbative $\mathcal{N}=4$ gauge theory and a topological string theory in twistor space.

The factorisation properties of amplitudes in the infrared play several roles in developing higher order perturbative predictions for observable quantities. First, a detailed knowledge of the structure of unresolved emission enables phase space integrations to be organised such that the infrared singularities due to soft or collinear emission can be analytically subtracted at NLO [48-50] or at NNLO [51]. Second, they enable large logarithmic corrections to be identified and resummed. Third, the collinear limit plays a crucial role in the unitarity-based method for loop calculations $[21,22,52,53]$.

In general, to compute a cross section at $\mathrm{N}^{n} \mathrm{LO}$, one requires detailed knowledge of the infrared factorisation functions describing the unresolved configurations for $n$-particles at tree-level, $(n-1)$-particles at one-loop etc. The universal behaviour in the double collinear limit is well known at tree-level (see for example Refs. [54, 55]), one-loop [21, 56-60] and at two-loops [61, 62]. Similarly, the triple collinear limit has 
been studied at tree-level [3-6] and, in the case of distinct quarks, at one-loop [63]. Finally, the tree-level quadruple gluon collinear limit was derived in Ref. [2, 7].

Our paper is organised as follows. In Section 2, we briefly review the colour ordered formalism that underpins the MHV rules. The relevant MHV vertices are given in Section 3. Section 4 describes the procedure for taking the collinear limit while the analytic structure of the splitting functions is discussed in Section $\mathbf{5}$. We write down general collinear factorization formulae in Section $\mathbf{6}$, which are valid for specific numbers of negative helicity partons and an arbitrary number of positive helicity partons. These results involve quarks and gluons in the collinear set and are complementary to the multi-gluon splitting functions derived in Ref. [2]. Specific explicit results for the collinear limits of up to three collinear partons are given in Sec. 7. Our findings are summarized in Sec. 8.

\section{Colour-ordered amplitudes}

Tree-level multi-particle amplitudes can be decomposed into colour-ordered partial amplitudes. For gluons only, this decomposition is given by

$$
\mathcal{A}_{n}\left(\left\{p_{i}, \lambda_{i}, a_{i}\right\}\right)=i g^{n-2} \sum_{\sigma \in S_{n} / Z_{n}} \operatorname{Tr}\left(T^{a_{\sigma(1)}} \cdots T^{a_{\sigma(n)}}\right) A_{n}\left(\sigma\left(1^{\lambda_{1}}, \ldots, n^{\lambda_{n}}\right)\right) .
$$

Here $S_{n} / Z_{n}$ is the group of non-cyclic permutations on $n$ symbols, and $j^{\lambda_{j}}$ labels the momentum $p_{j}$ and helicity $\lambda_{j}$ of the $j^{\text {th }}$ gluon, which carries the adjoint representation index $a_{i}$. The $T^{a_{i}}$ are fundamental representation $\mathrm{SU}\left(N_{c}\right)$ colour matrices, normalized so that $\operatorname{Tr}\left(T^{a} T^{b}\right)=\delta^{a b}$. The strong coupling constant is $\alpha_{s}=g^{2} /(4 \pi)$. Note that the MHV rules method of Ref. [1] is used to evaluate only the purely kinematic amplitudes $A_{n}$. Full amplitudes are then determined uniquely from the kinematic part $A_{n}$, and the known expressions for the colour traces.

For processes involving a quark-antiquark pair and an arbitrary number of gluons, the colour decomposition is given by

$$
\begin{aligned}
& \mathcal{A}_{n}\left(\left\{p_{i}, \lambda_{i}, a_{i}\right\},\left\{p_{j}, \lambda_{j}, i_{j}\right\}\right) \\
& \quad=i g^{n-2} \sum_{\sigma \in S_{n-2}}\left(T^{a_{\sigma(2)}} \cdots T^{a_{\sigma(n-1)}}\right)_{i_{1} i_{n}} A_{n}\left(1_{q}^{\lambda_{1}}, \sigma\left(2^{\lambda_{2}}, \ldots,(n-1)^{\lambda_{n-1}}\right), n_{\bar{q}}^{\lambda_{n}}\right),
\end{aligned}
$$

where $S_{n-2}$ is the set of permutations of $(n-2)$ gluons and the fermions carry the fundamental colour labels $i_{1}$ and $i_{n}$. By current conservation, the quark and antiquark helicities are related such that $\lambda_{1}=-\lambda_{n} \equiv \lambda$ where $\lambda= \pm \frac{1}{2}$.

When an additional photon with momentum $P_{\gamma}$ is emitted, the amplitudes have the following form,

$$
\begin{aligned}
& \mathcal{A}_{n}\left(\left\{p_{i}, \lambda_{i}, a_{i}\right\},\left\{p_{j}, \lambda_{j}, i_{j}\right\}, P_{\gamma}\right) \\
& \quad=i e g^{n-2} \sum_{\sigma \in S_{n-2}}\left(T^{a_{\sigma(2)}} \cdots T^{a_{\sigma(n-1)}}\right)_{i_{1} i_{n}} \tilde{A}_{n}\left(1_{q}^{\lambda_{1}}, \sigma\left(2^{\lambda_{2}}, \ldots,(n-1)^{\lambda_{n-1}}\right), n_{\bar{q}}^{\lambda_{n}} ; P_{\gamma}\right),
\end{aligned}
$$


where $e$ is the electric charge of the quark.

When there are two quark-antiquark pairs the tree-level amplitude can be decomposed into colour ordered amplitudes as,

$$
\begin{aligned}
& \mathcal{A}_{n}\left(\left\{p_{i}, \lambda_{i}, a_{i}\right\},\left\{p_{j}, \lambda_{j}, i_{j}\right\}\right)=i g^{n-2} \sum_{k}^{n-4} \sum_{\sigma \in S_{k}} \sum_{\rho \in S_{l}}\{ \\
& \left(T^{a_{\sigma(1)}} \cdots T^{a_{\sigma(k)}}\right)_{i_{1} i_{n}}\left(T^{a_{\rho(1)}} \cdots T^{a_{\rho(l)}}\right)_{i_{s+1} i_{s}} \\
& \left.\times A_{n}\left(1_{q}^{\lambda}, \sigma(1), \ldots, \sigma(k)\right), s_{\bar{Q}}^{-\lambda^{\prime}} ;(s+1)_{Q}^{\lambda^{\prime}}, \rho(1), \ldots, \rho(l), n_{\bar{q}}^{-\lambda}\right) \\
- & \frac{1}{N}\left(T^{a_{\sigma(1)}} \cdots T^{a_{\sigma(k)}}\right)_{i_{1} i_{s}}\left(T^{a_{\rho(1)}} \cdots T^{a_{\rho(l)}}\right)_{i_{s+1} i_{n}} \\
& \left.\times \tilde{A}_{n}\left(1_{q}^{\lambda}, \sigma(1), \ldots, \sigma(k), s_{\bar{q}}^{-\lambda} ;(s+1)_{Q}^{\lambda^{\prime}}, \rho(1), \ldots, \rho(l), n_{\bar{Q}}^{-\lambda^{\prime}}\right)\right\}
\end{aligned}
$$

where $S_{k}$ and $S_{l}$ are permutation groups such that $k+l=n-4$ and represent the possible ways of distributing the gluons in a colour ordered way between the quarks. For $i=j=0,\left(T^{a_{i}} \ldots T^{a_{j}}\right)_{k l}$ reduces to $\delta_{k l}$. We see that the two amplitudes $A_{n}$ and $\tilde{A}_{n}$ correspond to different ways of connecting the fundamental colour charges. For the $A$ amplitudes, there is a colour line connecting $q$ and $\bar{Q}$ and a second line connecting $Q$ and $\bar{q}$, while for the QED-like $\tilde{A}$ amplitudes the colour lines connect $q$ to $\bar{q}$ and $Q$ to $\bar{Q}$. Any number of gluons may be radiated from each colour line. As before, by current conservation, the quark and antiquark helicities are related such that $\lambda_{q}=-\lambda_{\bar{q}} \equiv \lambda$ and $\lambda_{Q}=-\lambda_{\bar{Q}} \equiv \lambda^{\prime}$ where $\lambda, \lambda^{\prime}= \pm \frac{1}{2}$.

\section{MHV amplitudes}

The colour ordered $n$-gluon MHV amplitude is given by

$$
A_{n}\left(1^{+}, \ldots, m_{1}^{-}, \ldots, m_{2}^{-}, \ldots, n^{+}\right)=\frac{\left\langle m_{1} m_{2}\right\rangle^{4}}{\prod_{i=1}^{n}\langle i i+1\rangle},
$$

while the two-quark multi-gluon MHV amplitudes are,

$$
A_{n}\left(1_{q}^{\lambda}, \ldots, m^{-}, \ldots, n_{\bar{q}}^{-\lambda}\right)=\frac{\langle m 1\rangle^{2-2 \lambda}\langle m n\rangle^{2+2 \lambda}}{\prod_{l=1}^{n}\langle l l+1\rangle} .
$$

Here the helicity of the quark is denoted by $\lambda= \pm \frac{1}{2}$ while ... denotes an arbitrary number of positive helicity gluons. Amplitudes for a quark-antiquark pair, many gluons and a photon are given by,

$$
\begin{aligned}
\tilde{A}\left(1_{q}^{\lambda}, \ldots, n_{\bar{q}}^{-\lambda} ; P_{\gamma}^{-}\right) & =\frac{\langle P 1\rangle^{2-2 \lambda}\langle P n\rangle^{2+2 \lambda}}{\langle P 1\rangle\langle 12\rangle \cdots\langle n P\rangle} \\
\tilde{A}\left(1_{q}^{\lambda}, \ldots, m^{-}, \ldots, n_{\bar{q}}^{-\lambda} ; P_{\gamma}^{+}\right) & =\frac{\langle m 1\rangle^{2-2 \lambda}\langle m n\rangle^{2+2 \lambda}}{\langle P 1\rangle\langle 12\rangle \cdots\langle n P\rangle}
\end{aligned}
$$


In the four-quark case, there are four MHV amplitudes where two of the fermions have negative helicity and two have positive helicity for each colour structure. For each helicity configuration we can write,

$$
\begin{aligned}
& A_{n}\left(1_{q}^{+}, \ldots, s_{\bar{Q}}^{-},(s+1)_{Q}^{+}, \ldots, n_{\bar{q}}^{-}\right)=\frac{\langle 1 s\rangle\langle s n\rangle^{2}\langle n s+1\rangle}{\prod_{l=1}^{n}\langle l l+1\rangle}, \\
& A_{n}\left(1_{q}^{+}, \ldots, s_{\bar{Q}}^{+},(s+1)_{Q}^{-}, \ldots, n_{\bar{q}}^{-}\right)=\frac{\langle 1 s\rangle\langle n s+1\rangle^{3}}{\prod_{l=1}^{n}\langle l l+1\rangle}, \\
& A_{n}\left(1_{q}^{-}, \ldots, s_{\bar{Q}}^{+},(s+1)_{Q}^{-}, \ldots, n_{\bar{q}}^{+}\right)=\frac{\langle 1 s\rangle\langle 1 s+1\rangle^{2}\langle n s+1\rangle}{\prod_{l=1}^{n}\langle l l+1\rangle}, \\
& A_{n}\left(1_{q}^{-}, \ldots, s_{\bar{Q}}^{-},(s+1)_{Q}^{+}, \ldots, n_{\bar{q}}^{+}\right)=\frac{\langle 1 s\rangle^{3}\langle n s+1\rangle}{\prod_{l=1}^{n}\langle l l+1\rangle}
\end{aligned}
$$

with the other colour ordering given by,

$$
\begin{aligned}
\tilde{A}_{n}\left(1_{q}^{+}, \ldots, s_{\bar{q}}^{-},(s+1)_{Q}^{+}, \ldots, n_{\bar{Q}}^{-}\right) & =\frac{\langle 1 n\rangle\langle n s\rangle^{2}\langle s s+1\rangle}{\prod_{l=1}^{n}\langle l l+1\rangle}, \\
\tilde{A}_{n}\left(1_{q}^{+}, \ldots, s_{\bar{q}}^{-},(s+1)_{Q}^{-}, \ldots, n_{\bar{Q}}^{+}\right) & =\frac{\langle 1 n\rangle\langle s s+1\rangle^{3}}{\prod_{l=1}^{n}\langle l l+1\rangle} \\
\tilde{A}_{n}\left(1_{q}^{-}, \ldots, s_{\bar{q}}^{+},(s+1)_{Q}^{-}, \ldots, n_{\bar{Q}}^{+}\right) & =\frac{\langle 1 n\rangle\langle 1 s+1\rangle^{2}\langle s s+1\rangle}{\prod_{l=1}^{n}\langle l l+1\rangle}, \\
\tilde{A}_{n}\left(1_{q}^{-}, \ldots, s_{\bar{q}}^{+},(s+1)_{Q}^{+}, \ldots, n_{\bar{Q}}^{-}\right) & =\frac{\langle 1 n\rangle^{3}\langle s s+1\rangle}{\prod_{l=1}^{n}\langle l l+1\rangle} .
\end{aligned}
$$

The $\overline{\mathrm{MHV}}$ amplitudes are related by parity and can be obtained by conjugating the MHV expressions,

$$
A_{n}\left(1^{\lambda_{1}}, \ldots, n^{\lambda_{n}}\right)=(-1)^{n}\left(A_{n}\left(1^{-\lambda_{1}}, \ldots, n^{-\lambda_{n}}\right)\right)^{*}
$$

and similarly for the $\tilde{A}$ amplitudes.

\section{Collinear limits}

To find the splitting functions we work with the colour stripped amplitudes. For these colour ordered amplitudes, it is known that when the collinear particles are not adjacent there is no collinear divergence [7]. Therefore, without loss of generality, we can take particles $1 \ldots n$ collinear.

The multiple collinear limit is approached when the momenta $p_{1}, \ldots, p_{n}$ become parallel. This implies that all the particle subenergies $s_{i j}=\left(p_{i}+p_{j}\right)^{2}$, with $i, j=$ $1, \ldots, n$, are simultaneously small. We thus introduce a pair of light-like momenta $P^{\nu}$ and $\xi^{\nu}\left(P^{2}=0, \xi^{2}=0\right)$, and we write

$$
\left(p_{1}+\cdots+p_{n}\right)^{\nu}=P^{\nu}+\frac{s_{1, n} \xi^{\nu}}{2 \xi \cdot P}, \quad s_{i, j}=\left(p_{i}+\cdots+p_{j}\right)^{2},
$$


where $s_{1, n}$ is the total invariant mass of the system of collinear partons. In the collinear limit, the vector $P^{\nu}$ denotes the collinear direction, and the individual collinear momenta are $p_{i}^{\nu} \rightarrow z_{i} P^{\nu}$. Here the longitudinal-momentum fractions $z_{i}$ are given by

$$
z_{i}=\frac{\xi \cdot p_{i}}{\xi \cdot P}
$$

and fulfil the constraint $\sum_{i=1}^{m} z_{i}=1$. To be definite, in the rest of the paper we work in the time-like region so that $\left(s_{i j}>0,1>z_{i}>0\right)$.

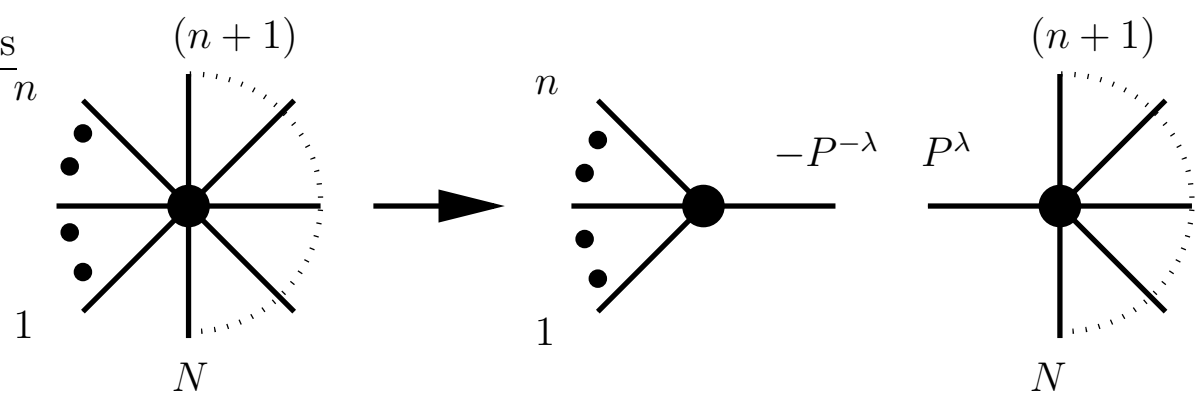

Figure 1: Factorisation of an $N$-point colour ordered amplitude with gluons $p_{1}, \ldots, p_{n}$ collinear into splitting function for $P \rightarrow 1, \ldots, n$ multiplied by an $(N-n+1)$-point amplitude.

As illustrated in Fig. 1, in the multi-collinear limit an $N$-particle colour ordered tree amplitude factorises and can be written as

$$
A_{N}\left(1^{\lambda_{1}}, \ldots, N^{\lambda_{N}}\right) \rightarrow \operatorname{split}\left(1^{\lambda_{1}}, \ldots, n^{\lambda_{n}} \rightarrow P^{\lambda}\right) \times A_{N-n+1}\left((n+1)^{\lambda_{n+1}}, \ldots, N^{\lambda_{N}}, P^{\lambda}\right)
$$

This labelling of the splitting amplitude $\operatorname{split}\left(1^{\lambda_{1}}, \ldots, n^{\lambda_{n}} \rightarrow P^{\lambda}\right)$ differs from the usual definition because we use the momentum and helicity that participates in the resultant amplitude $P^{\lambda}$ rather than $-P^{-\lambda}$. With this choice, it is easier to see how the helicity is conserved in the splitting, i.e. helicity $\lambda^{1}, \ldots, \lambda^{n}$ is replaced by $\lambda$. Since eq. (4.3) applies for all $N$, we can use it to derive the splitting amplitude by systematically choosing $N=3+n$. In this case, we always factorise onto a four-point amplitude.

\section{Analytic structure of splitting amplitudes}

The MHV rules of Ref. [1] were developed for calculating purely gluonic amplitudes at tree level and extended to amplitudes involving fermions in Ref. [12]. In this approach all non-MHV $N$-particle amplitudes (including $\overline{\mathrm{MHV}}$ ) are expressed as sums of tree diagrams in an effective scalar perturbation theory. The vertices in this 
theory are the MHV amplitudes of Eq. (1.1) continued off-shell and connected by scalar propagators $1 / q^{2}$.

Following [2], we classify collinear limits according to the difference between the number of negative helicity particles before taking the collinear limit, and the number after, $\Delta M$. Splitting amplitudes are calculated using the factorisation formula eq. (4.3). To facilitate the calculation, it makes sense to factorise onto hard amplitudes with the simplest analytic structure. Hence, in the MHV-rules formalism we will always factorise onto MHV amplitudes which are listed in section 3. In this case we find that $\Delta M$ of the splitting amplitude satisfies the relation,

$$
\Delta M+2=N_{-}
$$

where 2 is the number of negative helicities in the hard MHV amplitude, and $N_{-}$ is the total number of negative helicities in the full amplitude. $\Delta M$ determines the order of MHV diagram [1] for the full amplitude $A_{N}$

$$
\begin{aligned}
& \Delta M=0 \quad \Rightarrow \quad 1^{+}, 2^{+}, 3^{+}, \ldots, n^{+} \rightarrow P^{+} \quad A_{N}=\mathrm{MHV} \\
& 1^{-}, 2^{+}, 3^{+}, \ldots, n^{+} \rightarrow P^{-} \\
& \Delta M=1 \quad \Rightarrow \quad 1^{-}, 2^{+}, 3^{+}, \ldots, n^{+} \rightarrow P^{+} \quad A_{N}=\mathrm{NMHV} \\
& 1^{-}, 2^{-}, 3^{+}, \ldots, n^{+} \rightarrow P^{-} \\
& \Delta M=2 \quad \Rightarrow \quad 1^{-}, 2^{-}, 3^{+}, \ldots, n^{+} \rightarrow P^{+} \quad A_{N}=\mathrm{NNMHV} \\
& 1^{-}, 2^{-}, 3^{-}, \ldots, n^{+} \rightarrow P^{-}
\end{aligned}
$$

and so on for all $\Delta M>2$ cases.

If we choose to use $\overline{\mathrm{MHV}}$ rules, we extract the splitting function by factorising onto $\overline{\mathrm{MHV}}$ amplitudes. Splitting amplitudes are then classified by the difference in the number of positive helicity particles, $\Delta P$, and similar observations apply.

In general, any splitting amplitude can be obtained from either MHV or $\overline{\mathrm{MHV}}$ rules. A simple power counting argument [2] gives

$$
\text { split } \propto \frac{1}{[]^{\Delta M}\langle\rangle^{\Delta P}} .
$$

For an MHV-rules diagram to contribute to $\Delta M \neq 0$ collinear limits, it must contain anti-holomorphic spinor products $[i j]$ of collinear momenta. However, because onshell MHV vertices are entirely holomorphic, within the MHV rules there are only two potential sources of the anti-holomorphic spinor products. One source is scalar propagators $1 / s_{i j}=1 /\langle i j\rangle[j i]$ which connect MHV vertices. The second source is the off-shell continuation of the corresponding connected legs in the MHV vertices. 
Each off-shell continued leg of momentum $P$ gives rise to a factor $\langle i P\rangle \propto\langle i|P| \eta]$ which amounts to anti-holomorphic factors of the form $[j \eta]$. When the reference spinors $\eta_{\dot{\alpha}}$ are kept general, the $\eta$-dependence must cancel and therefore the off-shell continuation cannot give rise to an overall factor of $[i j]$.

This implies that within the MHV rules, the anti-holomorphic spinor products in (5.3) arise solely from the internal propagators. Since $\Delta M=v_{M H V}-1,{ }^{1}$ where $v_{M H V}$ is the number of MHV vertices in the diagram, the total number of internal propagators is $\Delta M$, in agreement with (5.3). Similarly, in the $\overline{\mathrm{MHV}}$ approach, the holomorphic products would arise solely from internal propagators whose total number in $\overline{\mathrm{MHV}}$ diagrams is $\Delta P$.

More precisely, it follows that all splitting amplitudes can be recast as

$$
\begin{aligned}
\text { split } & =\sum \frac{1}{\prod_{i, j=1}^{\Delta M} s_{i, j}} f(\langle\rangle) \\
& =\sum \frac{1}{\prod_{i, j=1}^{\Delta P} s_{i, j}} \tilde{f}([])
\end{aligned}
$$

where the first expression follows from the MHV rules representation, and the second expression - from the $\overline{\mathrm{MHV}}$ formalism. Here the summations are over all inequivalent choices of $\Delta M(\Delta P)$ products of vanishing kinematic invariants $s_{i, j}$ which corresponds to different MHV ( $\overline{\mathrm{MHV}})$ rules diagrams. The coefficient functions $f$ depend only on holomorphic spinor products, while the $\overline{\mathrm{MHV}}$ coefficients $\tilde{f}$ are purely anti-holomorphic. Moreover, $f$ and $\tilde{f}$ have dimensions,

$$
f \propto \frac{1}{\langle\rangle^{\Delta P-\Delta M}}, \quad \tilde{f} \propto \frac{1}{[]^{\Delta M-\Delta P}} .
$$

The fact that $f(\tilde{f})$ is purely (anti)-holomorphic suggests a simple twistor-space interpretation. All splitting functions can be represented as sums over the corresponding poles in $s$ with the coefficients being supported on a single degree-one curve in (anti)twistor space. This pure (anti)-holomorphic representation of multi-collinear limits is specific to the MHV ( $\overline{\mathrm{MHV}})$ formalism and is lost in the usual Feynman-diagramtype approaches as in Ref. [7], or in the BCF recursive approach, as shown in [2].

We further note that MHV rules for collinear limits are substantially simpler than the rules for the full amplitudes. Collinear splitting functions follow from a subset of the MHV rules diagrams [2]. The subset is determined by requiring that all internal propagators are on-shell in the multi-collinear limit. ${ }^{2}$ This is a powerful constraint on the types of the contributing diagrams and it simplifies taking the collinear limit dramatically.

\footnotetext{
${ }^{1}$ In principle, $\Delta M=v_{M H V}-v_{M H V}^{\prime}$ where $v_{M H V}^{\prime}$ is the number of MHV vertices remaining in the factored amplitude. The splitting function is independent of $v_{M H V}^{\prime}$, and because we systematically choose to factor directly onto a single MHV vertex, we set $v_{M H V}^{\prime}=1$.

${ }^{2}$ This is dictated by eq. (5.4) in the MHV formalism (or eq. (5.5) for $\overline{\mathrm{MHV}}$ rules).
} 
As mentioned earlier, each splitting amplitude can be calculated in both the MHV and in the $\overline{\mathrm{MHV}}$ approaches. In practice, eqs. (5.3)-(5.5) imply that the MHV approach is simpler if $\Delta M<\Delta P$, while the $\overline{\mathrm{MHV}}$ approach is more compact in the opposite case, $\Delta P<\Delta M$.

In most of what follows we will concentrate on the splitting amplitudes with $\Delta M \leq \Delta P$ and will follow the MHV rules. The remaining amplitudes with $\Delta P<$ $\Delta M$ are obtained from these by complex conjugation.

\subsection{An example}

When $\Delta M=\Delta P$ both $\mathrm{MHV}$ and $\overline{\mathrm{MHV}}$ rules are expected to yield results of similar complexity. As an example, let us consider a triple collinear splitting with $\Delta M=$ $\Delta P=1$. In full generality, the $\mathrm{MHV}(\overline{\mathrm{MHV}})$ rules approach should generate a maximum of three terms corresponding to simple poles in $s_{1,2}, s_{2,3}$ and $s_{1,3} \equiv\left(p_{1}+\right.$ $\left.p_{2}+p_{3}\right)^{2}$. For the specific splitting $1_{q}^{-}, 2_{\bar{Q}}^{+}, 3_{Q}^{-} \rightarrow P_{q}^{-}$, the MHV rules approach yields,

$$
\begin{aligned}
\operatorname{split}\left(1_{q}^{+}, 2_{\bar{Q}}^{+}, 3_{Q}^{-} \rightarrow P_{q}^{+}\right)= & -\frac{\langle 23\rangle z_{2}}{s_{2,3}\left(\langle 12\rangle \sqrt{z_{2}}+\langle 13\rangle \sqrt{z_{3}}\right)\left(z_{2}+z_{3}\right)} \\
& +\frac{\left(\langle 13\rangle \sqrt{z_{1}}+\langle 23\rangle \sqrt{z_{2}}\right)^{2}}{s_{1,3}\left(\langle 12\rangle \sqrt{z_{2}}+\langle 13\rangle \sqrt{z_{3}}\right)\langle 23\rangle},
\end{aligned}
$$

while the $\overline{\mathrm{MHV}}$ rules approach finds,

$$
\begin{aligned}
\operatorname{split}\left(1_{q}^{+}, 2_{\bar{Q}}^{+}, 3_{Q}^{-} \rightarrow P_{q}^{+}\right)= & \frac{z_{1} z_{3}[23]}{s_{2,3}\left([12] \sqrt{z_{2}}+[13] \sqrt{z_{3}}\right)\left(z_{2}+z_{3}\right)} \\
& -\frac{[12]^{2}}{s_{1,3}\left([12] \sqrt{z_{2}}+[13] \sqrt{z_{3}}\right)[23]} .
\end{aligned}
$$

As expected, the $s_{12}$ pole is absent because there is no $q \bar{Q}$ collinear limit. By taking the limit of a Feynman diagram calculation, Ref. [7] finds,

$$
\operatorname{split}\left(1_{q}^{+}, 2_{\bar{Q}}^{+}, 3_{Q}^{-} \rightarrow P_{q}^{+}\right)=\frac{\sqrt{z_{1} z_{2} z_{3}}}{s_{2,3}\left(z_{2}+z_{3}\right)}+\frac{[12]\left(\langle 13\rangle \sqrt{z_{1}}+\langle 23\rangle \sqrt{z_{2}}\right)}{s_{1,3} s_{2,3}} .
$$

Results (5.7), (5.8) and (5.9) are for the same amplitude and all three expressions agree numerically. But the analytic form of these specific representations is different. In agreement with eqs. (5.4)-(5.5), the functions accompanying the $1 / s$ poles, are holomorphic in the MHV result (5.7), are anti-holomorphic in the $\overline{\mathrm{MHV}}$ expression (5.8), while the Feynman diagram result (5.9) contains a mixture of holomorphic and anti-holomorphic terms. (In this case, it happens to give a more compact result.) In general, the limit of an amplitude computed using the BCF recursion relations will also provide a mixed holomorphic/anti-holomorphic splitting function (as discussed in sections $\mathbf{4 . 2 . 2}$ and $\mathbf{4} .2 .3$ of Ref. [2]). In this specific case, taking the collinear limit of the compact expression for the appropriate six-parton amplitude given in Ref. [41] exactly reproduces the MHV result of eq. (5.8). 
(a)

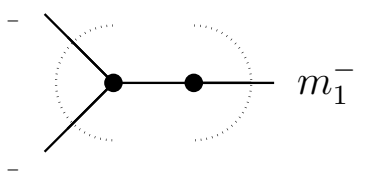

$(b)$

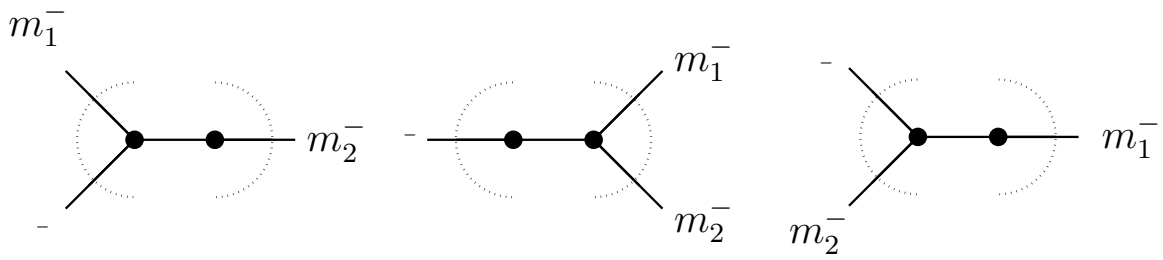

Figure 2: MHV topologies contributing to (a) $\operatorname{Split}_{+}\left(m_{1}\right)$ and (b) Split_ $\left(m_{1}, m_{2}\right)$. Negative helicity particles are indicated by solid lines, while arbitrary numbers of positive helicity particles emitted from each vertex are shown as dotted arcs. All particles that are not in the collinear set must be emitted from the left-hand vertex.

\section{General results}

In this section we give the results for the multiple collinear limit of quarks and gluons. We categorise the results according to the number of quarks involved in the limit. In each case, we give the general results for collinear limits with $\Delta M=0,1$ and involving an arbitrary number of positive helicity particles.

Limits of the type $\operatorname{split}\left(1^{+}, \ldots, n^{+} \rightarrow P^{+}\right)$and $\operatorname{split}\left(1^{-}, 2^{+}, \ldots, n^{+} \rightarrow P^{-}\right)$can contribute to the $\Delta M=0$, and these collinear splitting functions are straightforward to derive directly from the simple MHV vertex.

For the remaining splitting functions, it is useful to introduce the more compact notation

$$
\operatorname{split}\left(1^{+}, \ldots, m_{1}^{-}, \ldots, m_{2}^{-}, \ldots, m_{r}^{-}, \ldots, n^{+} \rightarrow P^{ \pm}\right)=\operatorname{Split}_{ \pm}\left(m_{1}, \ldots, m_{r}\right)
$$

For $\Delta M=1$, there are two possible types of splitting function, $\operatorname{Split}_{+}\left(m_{1}\right)$ and Split_ $\left(m_{1}, \ldots, m_{r}\right)$. The possible MHV topologies contributing to these splitting functions are illustrated in Fig. 2. Only negative helicity particles are shown. In the collinear limit, the propagator goes on-shell. Any MHV diagram with a hard particles emitted from both vertices produces an off-shell propagator. This means that only particles from the collinear set are allowed to couple to the right-hand vertex. All hard partons couple to the left-hand vertex.

Throughout we adopt the notation of Ref. [2]. In order that the limits can be read directly from the MHV diagrams, we make the following substitutions. If $a$ is a particle from the collinear set, $b$ is a particle which is not in the collinear set, and 
$q$ is the sum of the collinear momenta from $i+1$ to $j$, then

$$
\begin{aligned}
& \langle a q\rangle \rightarrow[P \eta] \sum_{l=i+1}^{j}\langle a l\rangle \sqrt{z_{l}} \equiv[P \eta] \Delta(i, j ; a), \\
& \langle b q\rangle \rightarrow[P \eta]\langle b P\rangle \sum_{l=i+1}^{j} z_{l}, \\
& \langle b a\rangle \rightarrow\langle b P\rangle \sqrt{z_{a}} .
\end{aligned}
$$

The $\Delta$ is defined as

$$
\Delta(i, j ; a)=\sum_{l=i+1}^{j}\langle a l\rangle \sqrt{z_{l}}
$$

noting that the boundary terms involving either $\langle 01\rangle$ or $\langle n n+1\rangle$, are given by,

$$
\begin{gathered}
\frac{\langle n n+1\rangle}{\Delta(i, n ; n+1)} \rightarrow-\frac{\sqrt{z_{n}}}{\sum_{l=i+1}^{n} z_{l}}, \\
\frac{\langle 01\rangle}{\Delta(0, j ; 0)} \rightarrow \frac{\sqrt{z_{1}}}{\sum_{k=1}^{j} z_{k}} .
\end{gathered}
$$

We also introduce

$$
D\left(i, j, q_{i+1, j}\right)=\frac{q_{i+1, j}^{2}}{\langle i, i+1\rangle\langle j, j+1\rangle} \Delta(i, j ; i) \Delta(i, j ; i+1) \Delta(i, j ; j) \Delta(i, j ; j+1) .
$$

\subsection{One quark in the collinear set: $q(n g) \rightarrow q$}

\subsection{1 $\Delta M=0$}

This is the simplest case which is read directly off the single MHV vertex. For positive helicity quarks, we use the two-quark MHV amplitude of Eq. (3.2) and find,

$$
\operatorname{split}\left(1_{q}^{+}, \ldots, n^{+} \rightarrow P_{q}^{+}\right)=\frac{\sqrt{z_{1}}}{\sqrt{z_{1} z_{n}} \prod_{l=1}^{n-1}\langle l, l+1\rangle}
$$

For negative helicity quarks,

$$
\operatorname{split}\left(1_{q}^{-}, \ldots, n^{+} \rightarrow P_{q}^{-}\right)=\frac{{\sqrt{z_{1}}}^{3}}{\sqrt{z_{1} z_{n}} \prod_{l=1}^{n-1}\langle l, l+1\rangle}
$$

Note that helicity conservation ensures that the helicity of $P$ is the same as that of $q$. It is often convenient to combine results for quarks of helicity $\lambda= \pm \frac{1}{2}$ such that,

$$
\operatorname{split}\left(1_{q}^{\lambda}, \ldots, n^{+} \rightarrow P_{q}^{\lambda}\right)=\frac{{\sqrt{z_{1}}}^{2-2 \lambda}}{\sqrt{z_{1} z_{n}} \prod_{l=1}^{n-1}\langle l, l+1\rangle}
$$


Using parity we find,

$$
\operatorname{split}\left(1_{q}^{+}, \ldots, n^{-} \rightarrow P_{q}^{+}\right)=\frac{(-1)^{n-1}{\sqrt{z_{1}}}^{2+2 \lambda}}{\sqrt{z_{1} z_{n}} \prod_{l=1}^{n-1}[l, l+1]} .
$$

The amplitudes where an antiquark is collinear with several gluons are obtained by charge conjugation.

\subsection{2 $\Delta M=1$}

Because of helicity conservation, $\Delta M=1$ implies that a single gluon has negative helicity. When the quark has positive helicity, then the MHV diagrams contributing in the collinear limit correspond to topology (a) of Fig. 2. There are two types of diagram - one class where the quark is emitted from the right-hand vertex (and the propagating particle is a quark) and one class mediated by gluon exchange where the quark is emitted from the left-hand vertex. We find,

$$
\begin{aligned}
\operatorname{split}\left(1_{q}^{+}, \ldots,\right. & \left.m^{-}, \ldots, n^{+} \rightarrow P_{q}^{+}\right)=\frac{1}{\sqrt{z_{1} z_{n}} \prod_{l=1}^{n-1}\langle l, l+1\rangle} \\
& \times\left[-\sum_{j=m}^{n} \frac{\Delta^{3}(0, j ; m)\langle 1 m\rangle}{D\left(0, j, q_{1, j}\right)}\left(\sum_{k=1}^{j} z_{k}\right)+\sum_{i=1}^{m-1} \sum_{j=m}^{n} \frac{\Delta^{4}(i, j ; m)}{D\left(i, j, q_{i+1, j}\right)} \sqrt{z_{1}}\right] .
\end{aligned}
$$

In the same manner, for negative helicity quarks, the allowed MHV diagrams correspond to the first and second topologies shown in Fig. 2(b),

$$
\begin{aligned}
\operatorname{split}\left(1_{q}^{-}, \ldots,\right. & \left.m^{-}, \ldots, n^{+} \rightarrow P_{q}^{-}\right)=\frac{1}{\sqrt{z_{1} z_{n}} \prod_{l=1}^{n-1}\langle l, l+1\rangle} \\
\times & {\left[-\sum_{j=m}^{n} \frac{\Delta(0, j ; m)\langle 1 m\rangle^{3}}{D\left(0, j, q_{1, j}\right)}\left(\sum_{k=1}^{j} z_{k}\right)^{3}+\sum_{i=1}^{m-1} \sum_{j=m}^{n} \frac{\Delta^{4}(i, j ; m)}{D\left(i, j, q_{i+1, j}\right)}{\sqrt{z_{1}}}^{3}\right] . }
\end{aligned}
$$

\subsection{Two quarks in the collinear set: $(n g) \bar{q} q \rightarrow g$}

In this collinear limit, the $\bar{q} q$ pair is in the adjoint representation and effectively acts as a gluon.

\subsection{1 $\Delta M=0$}

This is the simplest case which is read directly off the single MHV vertex. Unlike the previous case, here we start with a two-quark MHV amplitude and factorise onto a gluonic MHV amplitude. Alternatively, we could start with a four-quark amplitude and factorise onto a two-quark amplitude. For quarks with helicity $\lambda= \pm \frac{1}{2}$, we find,

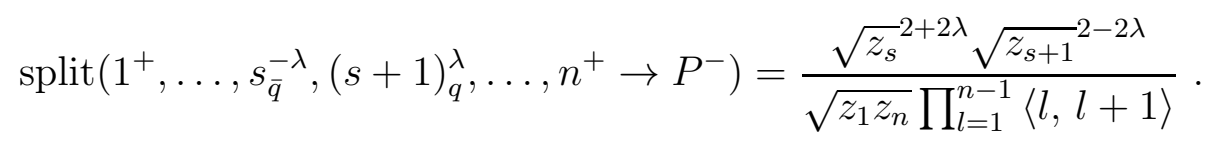


6.2.2 $\Delta M=1$

For amplitudes of the $\operatorname{Split}_{+}\left(m_{1}\right)$-type, we find

$$
\begin{aligned}
& \operatorname{split}\left(1^{+}, \ldots, s_{\bar{q}}^{-\lambda},(s+1)_{q}^{\lambda}, \ldots, n^{+} \rightarrow P^{+}\right)= \\
& \frac{1}{\sqrt{z_{1} z_{n}} \prod_{l=1}^{n-1}\langle l, l+1\rangle} \sum_{i=0}^{s-1} \sum_{j=s+1}^{n} \frac{\Delta^{2+2 \lambda}(i, j ; s) \Delta^{2-2 \lambda}(i, j ; s+1)}{D\left(i, j, q_{i+1, j}\right)} .
\end{aligned}
$$

There are four diagrams contributing to splitting functions of Split_ $\left(m_{1}, m_{2}\right)$ type ${ }^{3}$,

$$
\begin{aligned}
\operatorname{split}\left(1^{+}, \ldots, s_{\bar{q}}^{-\lambda},(s+1)_{q}^{\lambda}, \ldots,\right. & \left.m^{-}, \ldots, n^{+} \rightarrow P^{-}\right)=\frac{1}{\sqrt{z_{1} z_{n}} \prod_{l=1}^{n-1}\langle l, l+1\rangle} \\
& \times\left[\sum_{i=0}^{s-1} \sum_{j=m}^{n} \frac{\langle s m\rangle^{2+2 \lambda}\langle s+1 m\rangle^{2-2 \lambda}}{D\left(i, j, q_{i+1, j}\right)}\left(\sum_{k=i+1}^{j} z_{k}\right)^{4}\right. \\
& +\sum_{i=0}^{s-1} \sum_{j=s+1}^{m-1} \frac{\Delta^{2+2 \lambda}(i, j ; s) \Delta^{2-2 \lambda}(i, j ; s+1)}{D\left(i, j, q_{i+1, j}\right)} z_{m}^{2} \\
& -\sum_{j=m}^{n} \frac{\Delta^{2+2 \lambda}(s, j ; m)\langle s+1 m\rangle^{2-2 \lambda}}{D\left(s, j, q_{s+1, j}\right)}{\sqrt{z_{s}}}^{2+\lambda}\left(\sum_{k=s+1}^{j} z_{k}\right)^{2-2 \lambda} \\
& \left.+\sum_{i=s+1}^{m-1} \sum_{j=m}^{n} \frac{\Delta^{4}(i, j ; m)}{D\left(i, j, q_{i+1, j}\right)}{\sqrt{z_{s}}}^{2+2 \lambda}{\sqrt{z_{s+1}}}^{2-2 \lambda}\right] .
\end{aligned}
$$

Splitting functions of the type $\operatorname{split}\left(1^{+}, \ldots, m^{-}, \ldots, s_{\bar{q}}^{-\lambda},(s+1)_{q}^{\lambda}, \ldots, n^{+} \rightarrow P^{-}\right)$are obtained by line reversal. These results for the two-quark sector are sufficient to calculate all splitting amplitudes for up to four partons.

\subsection{Two quarks in the collinear set: $q(n g) \bar{q} \rightarrow \gamma$}

In this collinear limit, the $q \ldots \bar{q}$ system forms a colour singlet and effectively acts as a photon.

\subsection{1 $\Delta M=0$}

In this limit the four-quark $\tilde{A}$ MHV amplitudes of eqs. (3.9)-(3.12) factorise directly onto the two-quark+photon amplitudes of (3.3). We find that,

$$
\widetilde{\operatorname{split}}\left(1_{q}^{\lambda}, \ldots, n_{\bar{q}}^{-\lambda} \rightarrow P_{\gamma}^{-}\right)=\frac{z_{1}^{\frac{1}{2}-\lambda} z_{n}^{\frac{1}{2}+\lambda}}{\langle 12\rangle \cdots\langle n-1 n\rangle}
$$

${ }^{3}$ Diagrams where both the negative helicity fermion and gluon couple to the right-hand vertex in Fig. 2(b) can be mediated by either fermion or gluon exchange. 


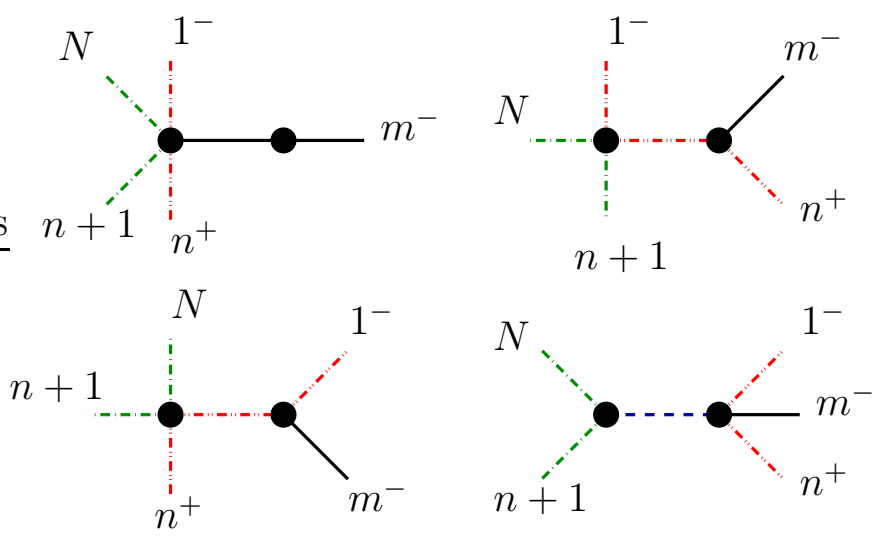

Figure 3: MHV topologies contributing to the two quark collinear limit of the type $\widehat{\operatorname{split}}\left(1_{q}^{-}, \ldots, m^{-}, \ldots, n_{\bar{q}}^{+} \rightarrow P_{\gamma}^{-}\right)$. Quarks of type $Q(q)$ are shown as green(red)-dotdashed lines and negative helicity gluons as black solid lines. The negative helicity photon is shown as a blue dashed line.

\subsection{2 $\Delta M=1$}

For amplitudes of the $\operatorname{Split}_{+}\left(m_{1}\right)$ type, there is a single MHV diagram and we find

$$
\widetilde{\operatorname{split}}\left(1_{q}^{-}, \ldots, n_{\bar{q}}^{+} \rightarrow P_{\gamma}^{+}\right)=\frac{1}{\sqrt{z_{1} z_{n}} \prod_{l=1}^{n-1}\langle l, l+1\rangle} \times \frac{\Delta(0, n ; 1)^{3} \Delta(0, n ; n)}{D\left(0, n ; q_{1, n}\right)}
$$

As in the previous case, there are four diagrams shown in Fig. 3 contributing to splitting functions of Split_ $\left(m_{1}, m_{2}\right)$ type such that,

$$
\begin{aligned}
\widetilde{\operatorname{split}}\left(1_{q}^{-}, \ldots,\right. & \left.m^{-}, \ldots, n_{\bar{q}}^{+} \rightarrow P_{\gamma}^{-}\right)=\frac{1}{\sqrt{z_{1} z_{n}} \prod_{l=1}^{n-1}\langle l, l+1\rangle} \\
& \times\left[\sum_{i=1}^{m-1} \sum_{j=m}^{n-1} \frac{\Delta(i, j ; m)^{4}{\sqrt{z_{1}}}^{3} \sqrt{z_{n}}}{D\left(i, j ; q_{i+1, j}\right)}-\sum_{i=1}^{m-1} \frac{\langle n m\rangle \Delta(i, n ; m)^{3}}{D\left(i, n ; q_{i+1, n}\right)}{\sqrt{z_{1}}}^{3}\left(\sum_{k=i+1}^{n} z_{k}\right)\right. \\
& \left.-\sum_{j=m}^{n-1} \frac{\langle 1 m\rangle^{3} \Delta(0, j ; m)}{D\left(0, j ; q_{1, j}\right)} \sqrt{z_{n}}\left(\sum_{k=1}^{j} z_{k}\right)^{3}+\frac{\langle 1 m\rangle^{3}\langle n m\rangle}{D\left(0, n ; q_{1, n}\right)}\right] .
\end{aligned}
$$

\subsection{Three quarks in the collinear set: $q(n g) \bar{Q} Q \rightarrow q$}

In this configuration, the $\bar{Q}$ is adjacent with $Q$ and therefore the vertices in the MHV rules include the four-quark amplitudes of eqs. (3.5)-(3.8). The factorised amplitude is a two-quark MHV as given in eq. (3.2) Furthermore, since the helicity of $q$ is conserved and the helicities of $Q$ and $\bar{Q}$ are opposite, there are no $\Delta M=0$ splitting functions. 


\subsection{1 $\Delta M=1$}

For $\Delta M=1$, the two diagrams (with quark and gluons exchanged) of $\operatorname{Split}_{+}\left(m_{1}\right)$ type yield,

$$
\begin{aligned}
& \operatorname{split}\left(1_{q}^{+}, \ldots, s_{\bar{Q}}^{-},(s+1)_{Q}^{+}\right.\left., \ldots, n^{+} \rightarrow P_{q}^{+}\right)=\frac{1}{\sqrt{z_{1} z_{n}} \prod_{l=1}^{n-1}\langle l, l+1\rangle} \\
& \times {\left[\sum_{i=1}^{s-1} \sum_{j=s+1}^{n} \frac{\Delta(i, j ; s+1) \Delta^{3}(i, j ; s)}{D\left(i, j, q_{i+1, j}\right)} \sqrt{z_{1}}\right.} \\
&\left.-\sum_{j=s+1}^{n} \frac{\langle 1 s\rangle \Delta^{2}(0, j ; s) \Delta(0, j ; s+1)}{D\left(0, j, q_{1, j}\right)}\left(\sum_{k=1}^{j} z_{k}\right)\right], \\
& \operatorname{split}\left(1_{q}^{+}, \ldots, s_{\bar{Q}}^{+},(s+1)_{Q}^{-}, \ldots, n^{+} \rightarrow P_{q}^{+}\right)=\frac{1}{\sqrt{z_{1} z_{n}} \prod_{l=1}^{n-1}\langle l, l+1\rangle} \\
& \times\left[\sum_{i=1}^{s-1} \sum_{j=s+1}^{n} \frac{\Delta^{3}(i, j ; s+1) \Delta(i, j ; s)}{D\left(i, j, q_{i+1, j}\right)} \sqrt{z_{1}}\right. \\
&\left.-\sum_{j=s+1}^{n} \frac{\langle 1 s\rangle \Delta^{3}(0, j ; s+1)}{D\left(0, j, q_{1, j}\right)}\left(\sum_{k=1}^{j} z_{k}\right)\right] .
\end{aligned}
$$

Similarly, the two diagrams of $\operatorname{Split}_{-}\left(m_{1}, m_{2}\right)$-type yield,

$$
\begin{aligned}
& \operatorname{split}\left(1_{q}^{-}, \ldots, s_{\bar{Q}}^{-},(s+1)_{Q}^{+}\right.\left., \ldots, n^{+} \rightarrow P_{q}^{-}\right)=\frac{1}{\sqrt{z_{1} z_{n}} \prod_{l=1}^{n-1}\langle l, l+1\rangle} \\
& \times {\left[\sum_{i=1}^{s-1} \sum_{j=s+1}^{n} \frac{\Delta(i, j ; s+1) \Delta^{3}(i, j ; s)}{D\left(i, j, q_{i+1, j}\right)}{\sqrt{z_{1}}}^{3}\right.} \\
&\left.-\sum_{j=s+1}^{n} \frac{\langle 1 s\rangle^{3} \Delta(0, j ; s+1)}{D\left(0, j, q_{1, j}\right)}\left(\sum_{k=1}^{j} z_{k}\right)^{3}\right] \\
& \operatorname{split}\left(1_{q}^{-}, \ldots, s_{\bar{Q}}^{+},(s+1)_{Q}^{-}, \ldots, n^{+} \rightarrow P_{q}^{-}\right)=\frac{1}{\sqrt{z_{1} z_{n}} \prod_{l=1}^{n-1}\langle l, l+1\rangle} \\
& \times\left[\sum_{i=1}^{s-1} \sum_{j=s+1}^{n} \frac{\Delta^{3}(i, j ; s+1) \Delta(i, j ; s)}{D\left(i, j, q_{i+1, j}\right)}{\sqrt{z_{1}}}^{3}\right. \\
&\left.-\sum_{j=s+1}^{n} \frac{\langle 1 s\rangle\langle 1 s+1\rangle^{2} \Delta(0, j ; s+1)}{D\left(0, j, q_{1, j}\right)}\left(\sum_{k=1}^{j} z_{k}\right)^{3}\right] .
\end{aligned}
$$

\subsection{Three quarks in the collinear set: $q(n g) \bar{q} Q \rightarrow Q$}

Here the relevant vertices in the MHV rules include the $\tilde{A}$ four-quark amplitudes of eqs. (3.9)-(3.12) and the factorised amplitude is a two-quark MHV as given in eq. (3.2) As in the previous case, the quark helicities are constrained such that there are no $\Delta M=0$ splitting functions. 
6.5.1 $\Delta M=1$

There are two diagrams for both $\operatorname{Split}_{+}\left(m_{1}\right)$ - and Split_ $\left(m_{1}, m_{2}\right)$-types and we find,

$$
\begin{aligned}
& \widetilde{\operatorname{split}}\left(1_{q}^{+}, \ldots, s_{\bar{q}}^{-},(s+1)_{Q}^{+}\right.\left., \ldots, n^{+} \rightarrow P_{Q}^{+}\right)=\frac{1}{\sqrt{z_{1} z_{n}} \prod_{l=1}^{n-1}\langle l, l+1\rangle} \\
& \times {\left[\frac{\Delta(0, s ; 1) \Delta^{3}(0, s ; s)}{D\left(0, s, q_{1, s}\right)} \sqrt{z_{s+1}}\right.} \\
&+\left.\sum_{j=s+1}^{n} \frac{\langle s s+1\rangle \Delta^{2}(0, j ; s) \Delta(0, j ; 1)}{D\left(0, j, q_{1, j}\right)}\left(\sum_{k=1}^{j} z_{k}\right)\right], \\
& \widetilde{\operatorname{split}}\left(1_{q}^{-}, \ldots, s_{\bar{q}}^{+},(s+1)_{Q}^{+}, \ldots, n^{+} \rightarrow P_{Q}^{+}\right)=\frac{1}{\sqrt{z_{1} z_{n}} \prod_{l=1}^{n-1}\langle l, l+1\rangle} \\
& \times\left[\frac{\Delta^{3}(0, s ; 1) \Delta(0, s ; s)}{D\left(0, s, q_{1, s}\right)} \sqrt{z_{s+1}}\right. \\
&+\left.\sum_{j=s+1}^{n} \frac{\langle s s+1\rangle \Delta^{3}(0, j ; 1)}{D\left(0, j, q_{1, j}\right)}\left(\sum_{k=1}^{j} z_{k}\right)\right]
\end{aligned}
$$

and,

$$
\begin{aligned}
& \widetilde{\operatorname{split}}\left(1_{q}^{+}, \ldots, s_{\bar{q}}^{-},(s+1)_{Q}^{-}, \ldots, n^{+} \rightarrow P_{Q}^{-}\right)=\frac{1}{\sqrt{z_{1} z_{n}} \prod_{l=1}^{n-1}\langle l, l+1\rangle} \\
& \times\left[\frac{\Delta^{3}(0, s ; 1) \Delta(0, s ; s)}{D\left(0, s, q_{1, s}\right)}{\sqrt{z_{s+1}}}^{3}\right. \\
& \left.+\sum_{j=s+1}^{n} \frac{\langle s s+1\rangle^{3} \Delta(0, j ; 1)}{D\left(0, j, q_{1, j}\right)}\left(\sum_{k=1}^{j} z_{k}\right)^{3}\right] \\
& \widetilde{\operatorname{split}}\left(1_{q}^{-}, \ldots, s_{\bar{q}}^{+},(s+1)_{Q}^{-}, \ldots, n^{+} \rightarrow P_{Q}^{-}\right)=\frac{1}{\sqrt{z_{1} z_{n}} \prod_{l=1}^{n-1}\langle l, l+1\rangle} \\
& \times\left[\frac{\Delta^{3}(0, s ; 1) \Delta(0, s ; s)}{D\left(0, s, q_{1, s}\right)}{\sqrt{z_{s+1}}}^{3}\right. \\
& \left.+\sum_{j=s+1}^{n} \frac{\langle s s+1\rangle\langle 1 s+1\rangle^{2} \Delta(0, j ; 1)}{D\left(0, j, q_{1, j}\right)}\left(\sum_{k=1}^{j} z_{k}\right)^{3}\right] \text {. }
\end{aligned}
$$

6.6 Four quarks in the collinear set: $\bar{Q} Q(n g) \bar{q} q \rightarrow g$

This limit is associated with the four-quark $A$-type colour ordered amplitude and is obtained by factoring onto a gluonic MHV.

\subsection{1 $\Delta M=1$}

Because of helicity conservation for the quarks, $\Delta M=0$ is forbidden. Furthermore, at least two negative helicity quarks participate in the scattering so that $\Delta M=1$ 


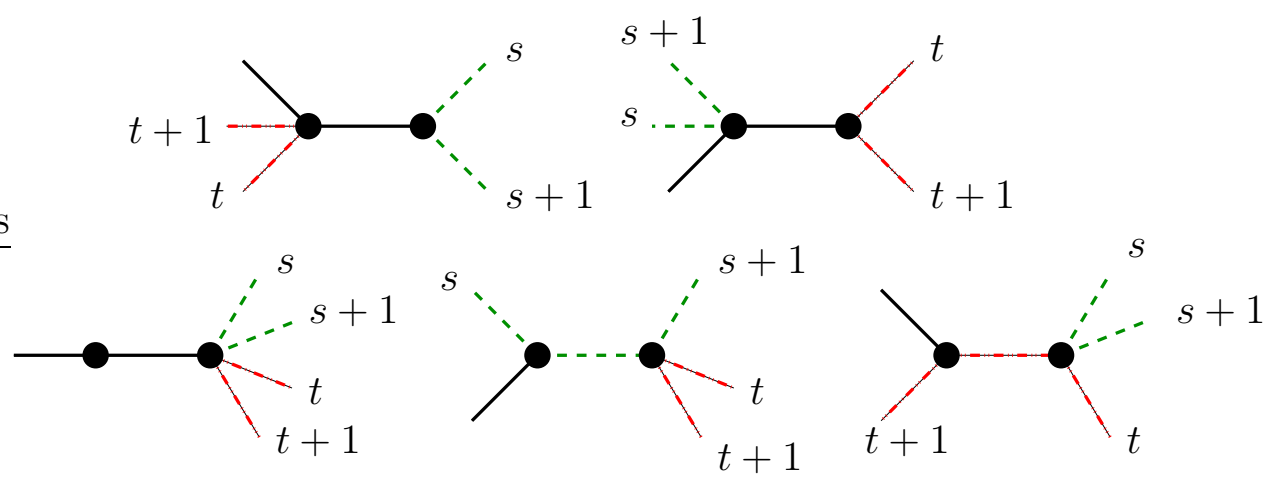

Figure 4: MHV topologies contributing to the four quark collinear limit of the type $\operatorname{split}\left(1^{+}, \ldots, s_{\bar{Q}}^{\lambda},(s+1)_{Q}^{-\lambda}, \ldots t_{\bar{q}}^{\lambda^{\prime}},(t+1)_{q}^{-\lambda^{\prime}}, \ldots, n^{+} \rightarrow P^{-}\right)$. Quarks of type $Q(q)$ are shown as green(red)-dotdashed lines and negative helicity gluons as black solid lines.

splittings must be of the Split_( $\left.m_{1}, m_{2}\right)$-type. The five contributing diagrams are shown in Fig. 4. Explicit evaluation of the four independent helicity configurations yields,

$$
\begin{aligned}
\operatorname{split}\left(1^{+}, \ldots, s_{\bar{Q}}^{+},(s+1)_{Q}^{-}\right. & \left., \ldots t_{\bar{q}}^{-},(t+1)_{q}^{+}, \ldots, n^{+} \rightarrow P^{-}\right)=\frac{1}{\sqrt{z_{1} z_{n}} \prod_{l=1}^{n-1}\langle l, l+1\rangle} \\
& \times\left[\sum_{i=0}^{s-1} \sum_{j=s+1}^{t-1} \frac{\Delta(i, j ; s) \Delta^{3}(i, j ; s+1)}{D\left(i, j, q_{i+1, j}\right)}{\sqrt{z_{t}}}^{3} \sqrt{z_{t+1}}\right. \\
& +\sum_{i=s+1}^{t-1} \sum_{j=t+1}^{n} \frac{\Delta^{3}(i, j ; t) \Delta(i, j ; t+1)}{D\left(i, j, q_{i+1, j}\right)}{\sqrt{z_{s}}{\sqrt{z_{s+1}}}^{3}}^{s-1}+\sum_{i=0} \sum_{j=t+1}^{n} \frac{\langle s+1 t\rangle^{3}\langle s t+1\rangle}{D\left(i, j, q_{i+1, j}\right)}\left(\sum_{k=i+1}^{j} z_{k}\right)^{4} \\
& -\sum_{j=t+1}^{n} \frac{\Delta(s, j ; t+1)\langle s+1 t\rangle^{3}}{D\left(s, j, q_{s+1, j}\right)} \sqrt{z_{s}}\left(\sum_{k=s+1}^{j} z_{k}\right)^{3} \\
& \left.+\sum_{i=0}^{s-1} \frac{\Delta(i, t ; s)\langle s+1 t\rangle^{3}}{D\left(i, t, q_{i+1, t}\right.} \sqrt{z_{t+1}}\left(\sum_{k=i+1}^{t} z_{k}\right)^{3}\right]
\end{aligned}
$$

$$
\begin{aligned}
\operatorname{split}\left(1^{+}, \ldots, s_{\bar{Q}}^{-},(s+1)_{Q}^{+}\right. & \left., \ldots t_{\bar{q}}^{-},(t+1)_{q}^{+}, \ldots, n^{+} \rightarrow P^{-}\right)=\frac{1}{\sqrt{z_{1} z_{n}} \prod_{l=1}^{n-1}\langle l, l+1\rangle} \\
& \times\left[\sum_{i=0}^{s-1} \sum_{j=s+1}^{t-1} \frac{\Delta^{3}(i, j ; s) \Delta(i, j ; s+1)}{D\left(i, j, q_{i+1, j}\right)}{\sqrt{z_{t}}}^{3} \sqrt{z_{t+1}}\right. \\
& +\sum_{i=s+1}^{t-1} \sum_{j=t+1}^{n} \frac{\Delta^{3}(i, j ; t) \Delta(i, j ; t+1)}{D\left(i, j, q_{i+1, j}\right)}{\sqrt{z_{s}}}^{3} \sqrt{z_{s+1}}
\end{aligned}
$$




$$
\begin{aligned}
& +\sum_{i=0}^{s-1} \sum_{j=t+1}^{n} \frac{\langle s+1 t\rangle\langle s t+1\rangle\langle t s\rangle^{2}}{D\left(i, j, q_{i+1, j}\right)}\left(\sum_{k=i+1}^{j} z_{k}\right)^{4} \\
& -\sum_{j=t+1}^{n} \frac{\Delta(s, j ; t+1) \Delta^{2}(s, j ; t)\langle s+1 t\rangle}{D\left(s, j, q_{s+1, j}\right)}{\sqrt{z_{s}}}^{3}\left(\sum_{k=s+1}^{j} z_{k}\right) \\
& \left.+\sum_{i=0}^{s-1} \frac{\Delta(i, t ; s)\langle s+1 t\rangle\langle t s\rangle^{2}}{D\left(i, t, q_{i+1, t}\right)} \sqrt{z_{t+1}}\left(\sum_{k=i+1}^{t} z_{k}\right)^{3}\right]
\end{aligned}
$$

$\operatorname{split}\left(1^{+}, \ldots, s_{\bar{Q}}^{+},(s+1)_{Q}^{-}, \ldots t_{\bar{q}}^{+},(t+1)_{q}^{-}, \ldots, n^{+} \rightarrow P^{-}\right)=\frac{1}{\sqrt{z_{1} z_{n}} \prod_{l=1}^{n-1}\langle l, l+1\rangle}$

$$
\begin{aligned}
& \times\left[\sum_{i=0}^{s-1} \sum_{j=s+1}^{t-1} \frac{\Delta(i, j ; s) \Delta^{3}(i, j ; s+1)}{D\left(i, j, q_{i+1, j}\right)}{\sqrt{z_{t}}}^{z_{t+1}}{ }^{3}\right. \\
& +\sum_{i=s+1}^{t-1} \sum_{j=t+1}^{n} \frac{\Delta(i, j ; t) \Delta^{3}(i, j ; t+1)}{D\left(i, j, q_{i+1, j}\right)}{\sqrt{z_{s}}}^{z_{s+1}}{ }^{3} \\
& +\sum_{i=0}^{s-1} \sum_{j=t+1}^{n} \frac{\langle s+1 t\rangle\langle s t+1\rangle\langle s+1 t+1\rangle^{2}}{D\left(i, j, q_{i+1, j}\right)}\left(\sum_{k=i+1}^{j} z_{k}\right)^{4} \\
& -\sum_{j=t+1}^{n} \frac{\Delta(s, j ; t+1)\langle s+1 t\rangle\langle s+1 t+1\rangle^{2}}{D\left(s, j, q_{s+1, j}\right)}{\sqrt{z_{s}}}^{s}\left(\sum_{k=s+1}^{j} z_{k}\right)^{3} \\
& \left.+\sum_{i=0}^{s-1} \frac{\Delta(i, t ; s) \Delta^{2}(i, t ; s+1)\langle s+1 t\rangle}{D\left(i, t, q_{i+1, t}\right)}{\sqrt{z_{t+1}}}^{t}\left(\sum_{k=i+1}^{t} z_{k}\right)\right]
\end{aligned}
$$

$$
\begin{aligned}
\operatorname{split}\left(1^{+}, \ldots, s_{\bar{Q}}^{-},(s+1)_{Q}^{+}\right. & \left., \ldots t_{\bar{q}}^{+},(t+1)_{q}^{-}, \ldots, n^{+} \rightarrow P^{-}\right)=\frac{1}{\sqrt{z_{1} z_{n}} \prod_{l=1}^{n-1}} \\
& \times\left[\sum_{i=0}^{s-1} \sum_{j=s+1}^{t-1} \frac{\Delta^{3}(i, j ; s) \Delta(i, j ; s+1)}{D\left(i, j, q_{i+1, j}\right)}{\sqrt{z_{t}}{\sqrt{z_{t+1}}}^{3}}^{t-1} \sum_{i=s+1}^{n} \sum_{j=t+1}^{n} \frac{\Delta(i, j ; t) \Delta^{3}(i, j ; t+1)}{D\left(i, j, q_{i+1, j}\right)}{\sqrt{z_{s}}}^{3}{\sqrt{z_{s+1}}}^{s-1}{ }^{n} \sum_{i=0}^{n} \sum_{j=t+1} \frac{\langle s+1 t\rangle\langle s t+1\rangle^{3}}{D\left(i, j, q_{i+1, j}\right)}\left(\sum_{k=i+1}^{j} z_{k}\right)^{4}\right. \\
& -\sum_{j=t+1}^{n} \frac{\Delta^{3}(s, j ; t+1)\langle s+1 t\rangle}{D\left(s, j, q_{s+1, j}\right)}{\sqrt{z_{s}}}^{3}\left(\sum_{k=s+1}^{j} z_{k}\right) \\
& \left.+\sum_{i=0}^{s-1} \frac{\Delta^{3}(i, t ; s)\langle s+1 t\rangle}{D\left(i, t, q_{i+1, t}\right)}{\sqrt{z_{t+1}}}^{3}\left(\sum_{k=i+1}^{t} z_{k}\right)\right] .
\end{aligned}
$$




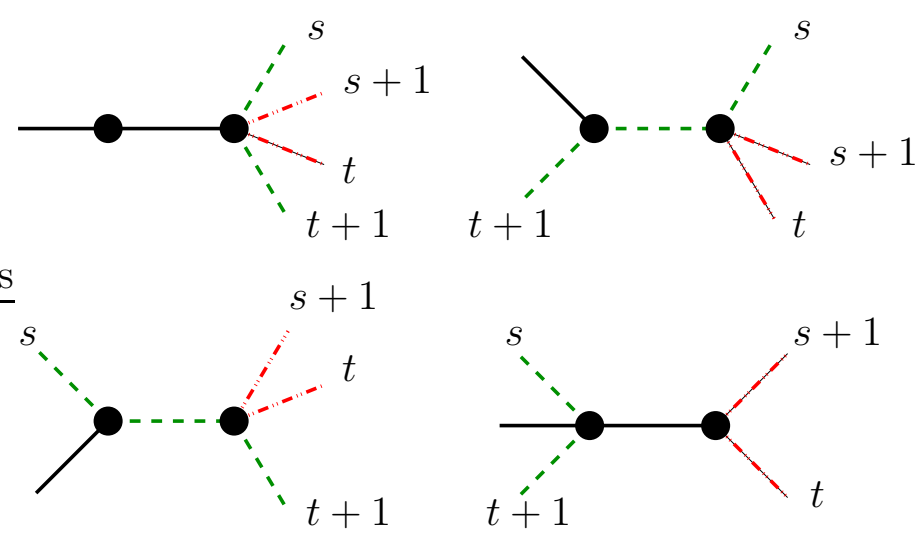

Figure 5: MHV topologies contributing to the four quark collinear limit of the type $\widetilde{\operatorname{split}}\left(1^{+}, \ldots, s_{\bar{Q}}^{\lambda},(s+1)_{q}^{-\lambda^{\prime}}, \ldots t_{\bar{q}}^{\lambda^{\prime}},(t+1)_{Q}^{-\lambda}, \ldots, n^{+} \rightarrow P^{-}\right)$. Quarks of type $Q(q)$ are shown as green(red)-dotdashed lines and negative helicity gluons as black solid lines.

\subsection{Four quarks in the collinear set: $\bar{Q} q(n g) \bar{q} Q \rightarrow g$}

This limit is associated with the four-quark $\tilde{A}$-type colour ordered amplitude and is obtained by factoring onto a gluonic MHV.

\subsection{1 $\Delta M=1$}

As in the previous case, helicity conservation for the quarks, ensures that $\Delta M=0$ is forbidden and that $\operatorname{Split}_{+}\left(m_{1}\right)$-type $\Delta M=1$ splittings are absent. The four contributing diagrams of Split_ $\left(m_{1}, m_{2}\right)$-type are shown in Fig. 5.

The four independent helicity configurations are given by,

$$
\begin{aligned}
\widetilde{\operatorname{split}}\left(1^{+}, \ldots, s_{\bar{Q}}^{+},(s+1)_{q}^{+}, \ldots t_{\bar{q}}^{-},(t+1)_{Q}^{-}, \ldots, n^{+} \rightarrow P^{-}\right)=\frac{1}{\sqrt{z_{1} z_{n}} \prod_{l=1}^{n-1}\langle l, l+1\rangle} \\
\times\left[\sum_{i=0}^{s-1} \sum_{j=t+1}^{n} \frac{\langle t+1 t\rangle^{3}\langle s s+1\rangle}{D\left(i, j, q_{i+1, j}\right)}\left(\sum_{k=i+1}^{j} z_{k}\right)^{4}\right. \\
-\sum_{j=t+1}^{n} \frac{\Delta(s, j ; s+1)\langle t+1 t\rangle^{3}}{D\left(s, j, q_{s+1, j}\right)} \sqrt{z_{s}}\left(\sum_{k=s+1}^{j} z_{k}\right)^{3} \\
-\sum_{i=0}^{s-1} \frac{\Delta^{3}(i, t ; t)\langle s s+1\rangle}{D\left(i, t, q_{i+1, t}\right)} \sqrt{z_{t+1}} 3\left(\sum_{k=i+1}^{t} z_{k}\right)^{3}, \\
\left.+\frac{\Delta^{3}(s, t ; t) \Delta(s, t ; s+1)}{D\left(s, t, q_{s+1, t}\right)} \sqrt{z_{s}} \sqrt{z_{t+1}}\right]
\end{aligned}
$$

$$
\begin{array}{r}
\widetilde{\operatorname{split}}\left(1^{+}, \ldots, s_{\bar{Q}}^{+},(s+1)_{q}^{-}, \ldots t_{\bar{q}}^{+},(t+1)_{Q}^{-}, \ldots, n^{+} \rightarrow P^{-}\right)=\frac{1}{\sqrt{z_{1} z_{n}} \prod_{l=1}^{n-1}\langle l, l+1\rangle} \\
\times\left[\sum_{i=0}^{s-1} \sum_{j=t+1}^{n} \frac{\langle t+1 t\rangle\langle s s+1\rangle\langle s+1 t+1\rangle^{2}}{D\left(i, j, q_{i+1, j}\right)}\left(\sum_{k=i+1}^{j} z_{k}\right)^{4}\right.
\end{array}
$$




$$
\begin{aligned}
& -\sum_{j=t+1}^{n} \frac{\Delta(s, j ; s+1)\langle t+1 t\rangle\langle s+1 t+1\rangle^{2}}{D\left(s, j, q_{s+1, j}\right)} \sqrt{z_{s}}\left(\sum_{k=s+1}^{j} z_{k}\right)^{3} \\
& -\sum_{i=0}^{s-1} \frac{\Delta(i, t ; t) \Delta^{2}(i, t ; s+1)\langle s s+1\rangle}{D\left(i, t, q_{i+1, t}\right)}{\sqrt{z_{t+1}}}^{3}\left(\sum_{k=i+1}^{t} z_{k}\right) \\
& \left.+\frac{\Delta(s, t ; t) \Delta^{3}(s, t ; s+1)}{D\left(s, t, q_{s+1, t}\right)} \sqrt{z_{s}}{\sqrt{z_{t+1}}}^{3}\right],
\end{aligned}
$$

$\widetilde{\operatorname{split}}\left(1^{+}, \ldots, s_{\bar{Q}}^{-},(s+1)_{q}^{+}, \ldots t_{\bar{q}}^{-},(t+1)_{Q}^{+}, \ldots, n^{+} \rightarrow P^{-}\right)=\frac{1}{\sqrt{z_{1} z_{n}} \prod_{l=1}^{n-1}\langle l, l+1\rangle}$

$$
\times\left[\sum_{i=0}^{s-1} \sum_{j=t+1}^{n} \frac{\langle t+1 t\rangle\langle s s+1\rangle\langle t s\rangle^{2}}{D\left(i, j, q_{i+1, j}\right)}\left(\sum_{k=i+1}^{j} z_{k}\right)^{4}\right.
$$$$
-\sum_{j=t+1}^{n} \frac{\Delta(s, j ; s+1) \Delta^{2}(s, j ; t)\langle t+1 t\rangle}{D\left(s, j, q_{s+1, j}\right)}{\sqrt{z_{s}}}^{3}\left(\sum_{k=s+1}^{j} z_{k}\right)
$$$$
-\sum_{i=0}^{s-1} \frac{\Delta(i, t ; t)\langle s s+1\rangle\langle t s\rangle^{2}}{D\left(i, t, q_{i+1, t}\right)} \sqrt{z_{t+1}}\left(\sum_{k=i+1}^{t} z_{k}\right)^{3}
$$

$$
\begin{aligned}
& \left.+\frac{\Delta^{3}(s, t ; t) \Delta(s, t ; s+1)}{D\left(s, t, q_{s+1, t}\right)}{\sqrt{z_{s}}}^{3} \sqrt{z_{t+1}}\right], \\
\widetilde{\operatorname{split}}\left(1^{+}, \ldots, s_{\bar{Q}}^{-},(s+1)_{q}^{-}, \ldots t_{\bar{q}}^{+}\right. & \left.(t+1)_{Q}^{+}, \ldots, n^{+} \rightarrow P^{-}\right)=\frac{1}{{\sqrt{z_{1} z_{n}} \prod_{l=1}^{n-1}\langle l, l}^{4}} \\
& \times\left[\sum_{i=0}^{s-1} \sum_{j=t+1}^{n} \frac{\langle t+1 t\rangle\langle s s+1\rangle^{3}}{D\left(i, j, q_{i+1, j}\right)}\left(\sum_{k=i+1}^{j} z_{k}\right)^{j}\right. \\
& -\sum_{j=t+1}^{n} \frac{\Delta^{3}(s, j ; s+1)\langle t+1 t\rangle}{D\left(s, j, q_{s+1, j}\right)}{\sqrt{z_{s}}}^{3}\left(\sum_{k=s+1}^{j} z_{k}\right) \\
& \left.-\sum_{i=0}^{s-1} \frac{\Delta(i, t ; t)\langle s s+1\rangle^{3}}{D\left(i, t, q_{i+1, t}\right)}{\sqrt{z_{t+1}}}^{3} \sum_{k=i+1}^{t} z_{k}\right)^{3} \\
& +\frac{\Delta(s, t ; t) \Delta^{3}(s, t ; s+1)}{D\left(s, t, q_{s+1, t}\right)}{\sqrt{z_{s}}}^{3}{\sqrt{z_{t+1}}} .
\end{aligned}
$$

\section{Selected results for triple collinear limits}

To illustrate our general results for multi-collinear limits, in this section we list some of the triple-collinear splitting functions. The $\Delta M=0$ splitting amplitudes are obtained directly from MHV amplitudes and we do not list them here. Explicit results are given in Section 6 . For the $\Delta M=1$ (and therefore $\Delta P=1$ ) amplitudes, there are two types of splitting function corresponding to $\operatorname{Split}_{+}\left(m_{1}\right)$ and $\operatorname{Split}_{-}\left(m_{1}, m_{2}\right)$. In the specific case of three collinear particles, these are related by parity thereby 
reducing the number of independent amplitudes to at most two for each splitting. Here, we list only the most compact form of the amplitudes.

\section{$7.1 q g g \rightarrow q$}

There are only two independent $\Delta M=1$ splitting amplitudes, which can be obtained by setting $m=n=3$ in Eqs. (6.13) and (6.14). Explicitly we find,

$$
\begin{aligned}
\operatorname{split}\left(1_{q}^{+}, 2^{+}, 3^{-} \rightarrow P_{q}^{+}\right)= & -\frac{\langle 23\rangle z_{2}^{3 / 2}}{\sqrt{z_{3}} s_{2,3}\left(\langle 21\rangle \sqrt{z_{2}}+\langle 31\rangle \sqrt{z_{3}}\right)\left(z_{2}+z_{3}\right)} \\
& -\frac{\langle 13\rangle\left(\langle 13\rangle \sqrt{z_{1}}+\langle 23\rangle \sqrt{z_{2}}\right)^{2}}{\langle 12\rangle\langle 23\rangle s_{1,3}\left(\langle 21\rangle \sqrt{z_{2}}+\langle 31\rangle \sqrt{z_{3}}\right)}, \\
\operatorname{split}\left(1_{q}^{-}, 2^{+}, 3^{-} \rightarrow P_{q}-\right)= & -\frac{z_{1}\langle 23\rangle z_{2}^{3 / 2}}{\sqrt{z_{3}} s_{2,3}\left(\langle 21\rangle \sqrt{z_{2}}+\langle 31\rangle \sqrt{z_{3}}\right)\left(z_{2}+z_{3}\right)} \\
& -\frac{\langle 13\rangle^{3}}{\langle 12\rangle\langle 23\rangle s_{1,3}\left(\langle 21\rangle \sqrt{z_{2}}+\langle 31\rangle \sqrt{z_{3}}\right)} .
\end{aligned}
$$

All others can be obtained by parity and charge conjugation. These expressions numerically agree with the splitting functions given in $[7]^{4}$,

$$
\begin{aligned}
\operatorname{split}\left(1_{q}^{+}, 2^{+}, 3^{-} \rightarrow P_{q}^{+}\right)= & -\frac{1}{s_{1,2} s_{2,3}} \\
\times & {\left[\frac{[12]\left(\langle 13\rangle \sqrt{z_{1}}+\langle 23\rangle \sqrt{z_{2}}\right)^{2}\left([12] \sqrt{z_{1}}+[32] \sqrt{z_{3}}\right)}{s_{1,3}}\right.} \\
& +\frac{\sqrt{z_{2}}\left(z_{1}+z_{2}\right)[12]\left(\langle 13\rangle \sqrt{z_{1}}+\langle 23\rangle \sqrt{z_{2}}\right)}{\sqrt{z_{3}}} \\
& \left.+\frac{\sqrt{z_{1}} z_{2} s_{1,2}}{\left(z_{2}+z_{3}\right)}\right], \\
\operatorname{split}\left(1_{q}^{-}, 2^{+}, 3^{-} \rightarrow P_{q}-\right)= & -\frac{1}{s_{1,2} s_{2,3}} \\
\times & {\left[\frac{[13]\left(\langle 12\rangle \sqrt{z_{1}}+\langle 32\rangle \sqrt{z_{3}}\right)^{2}\left([13] \sqrt{z_{1}}+[23] \sqrt{z_{2}}\right)}{s_{1,3}}\right.} \\
& +\frac{\sqrt{z_{2}}\left(z_{1}+z_{2}\right)[13]\left(\langle 12\rangle \sqrt{z_{1}}+\langle 32\rangle \sqrt{z_{3}}\right)}{\sqrt{z_{3}}} \\
& \left.+\frac{\sqrt{z_{1}} z_{2} s_{1,2}}{\left(z_{2}+z_{3}\right)}+\sqrt{z_{2}}\langle 23\rangle[13]\right] .
\end{aligned}
$$

We see that the two sets of results have the same types of singularity structure as $z_{3} \rightarrow 0$ and $z_{1} \rightarrow 1$ corresponding to the soft and double soft gluon limits.

\footnotetext{
${ }^{4}$ Note that there is a small typographical error in Eq. (5.56) of Ref. [7]. $s_{23}$ should be replaced by $s_{12}$ in the last term of the equation for $\operatorname{split}_{-}^{q \rightarrow q g g}\left(k_{1}^{+}, k_{2}^{+}, k_{3}^{-}\right)$
} 


\section{$7.2 \bar{q} q g \rightarrow g$}

There are again only two independent $\Delta M=1$ amplitudes, both of which can be obtained from Eq. (6.16) by setting $s=2$ and $\lambda= \pm \frac{1}{2}$. We find,

$$
\begin{aligned}
\operatorname{split}\left(1^{+}, 2_{\bar{q}}^{+}, 3_{q}^{-} \rightarrow P^{+}\right)= & -\frac{\left(\langle 12\rangle \sqrt{z_{1}}+\langle 32\rangle \sqrt{z_{3}}\right)\left(\langle 13\rangle \sqrt{z_{1}}+\langle 23\rangle \sqrt{z_{2}}\right)^{2}}{\langle 12\rangle\langle 23\rangle s_{1,3}\left(\langle 21\rangle \sqrt{z_{2}}+\langle 31\rangle \sqrt{z_{3}}\right)} \\
& +\frac{z_{2}\langle 23\rangle}{\sqrt{z_{1}} s_{2,3}\left(\langle 21\rangle \sqrt{z_{2}}+\langle 31\rangle \sqrt{z_{3}}\right)\left(z_{2}+z_{3}\right)}, \\
\operatorname{split}\left(1^{+}, 2_{\bar{q}}^{-}, 3_{q}^{+} \rightarrow P^{+}\right)= & -\frac{\left(\langle 12\rangle \sqrt{z_{1}}+\langle 32\rangle \sqrt{z_{3}}\right)^{3}}{\langle 12\rangle\langle 23\rangle s_{1,3}\left(\langle 21\rangle \sqrt{z_{2}}+\langle 31\rangle \sqrt{z_{3}}\right)} \\
& +\frac{z_{3}\langle 23\rangle}{\sqrt{z_{1}} s_{2,3}\left(\langle 21\rangle \sqrt{z_{2}}+\langle 31\rangle \sqrt{z_{3}}\right)\left(z_{2}+z_{3}\right)} .
\end{aligned}
$$

All others can be obtained via parity and charge conjugation. Eq. (7.5) numerically agrees with the analogous expression given in Ref. [7]. We were not able to find agree-

ment between Eq. (7.6) and the expression for $\operatorname{split}_{-}^{g \rightarrow g q \bar{q}}\left(k_{1}^{+}, k_{2}^{-}, k_{3}^{+}\right)$in Eq. (5.53) of Ref. [7].

\section{$7.3 q \bar{Q} Q \rightarrow q$ and $q \bar{q} Q \rightarrow Q$}

In this special case both colour structures lead to the same splitting amplitude. There is only one independent $\Delta M=1$ helicity configurations. It is given in Eq. (5.7).

\section{Conclusion}

In this paper we have considered the collinear limit of multi-parton QCD amplitudes at tree level. We have used the new MHV rules for constructing colour ordered amplitudes from MHV vertices to generalise our previous results for gluon-only splitting functions. Our main results are general formulae for timelike splitting functions involving up to two negative helicity partons and an arbitrary number of positive helicity partons. We anticipate that the expressions presented here will be useful in developing higher order perturbative predictions for observable quantities, such as jet cross sections at the LHC or in examining the high energy limit of QCD.

A key point of our approach is that in the collinear limit only a subset of MHV rules diagrams contribute - those where every propagator invariant $s$ goes on-shell in the multi-collinear limit. We observe that the splitting functions have a simple structure, and can be written as sums over the corresponding poles in $s$ multiplied by a coefficient that is either entirely composed of holomorphic spinor products $\langle i j\rangle$ or entirely composed of anti-holomorphic spinor products $[i j]$. This implies that the coefficients are supported on a single degree-one curve in (anti)-twistor space. 


\section{Acknowledgements}

EWNG and VVK acknowledge the support of PPARC through Senior Fellowships and TGB acknowledges the award of a PPARC studentship.

\section{References}

[1] F. Cachazo, P. Svrček and E. Witten, MHV vertices and tree amplitudes in gauge theory, JHEP 09 (2004) 006 [arXiv:hep-th/0403047].

[2] T.G. Birthwright, E.W.N. Glover, V.V. Khoze and P. Marquard, Multi-gluon collinear limits from MHV diagrams, JHEP 05 (2005) 013 [arXiv:hep-ph/0503063].

[3] A. Gehrmann-De Ridder and E.W.N. Glover, A complete $\mathcal{O}\left(\alpha \alpha_{s}\right)$ calculation of the photon +1 jet rate in $e^{+} e^{-}$annihilation, Nucl. Phys. B517 (1998) 269 hep-ph/9707224.

[4] J.M. Campbell and E.W.N. Glover, Double unresolved approximations to multiparton scattering amplitudes, Nucl. Phys. B527 (1998) 264 hep-ph/9710255.

[5] S. Catani and M. Grazzini, Collinear factorization and splitting functions for next-to-next-to-leading order QCD calculations, Phys. Lett. B446 (1999) 143 hep-ph/9810389.

[6] S. Catani and M. Grazzini, Infrared factorization of tree level QCD amplitudes at the next-to-next-to-leading order and beyond, Nucl. Phys. B570 (2000) 287 hep-ph/9908523.

[7] V. Del Duca, A. Frizzo and F. Maltoni, Factorization of tree QCD amplitudes in the high-energy limit and in the collinear limit, Nucl. Phys. B568, 211 (2000) hep-ph/9909464.

[8] D.A. Kosower, All-order collinear behavior in gauge theories, Nucl. Phys. B552 (1999) 319 hep-ph/9901201.

[9] S.J. Parke and T.R. Taylor, An amplitude for n-gluon scattering, Phys. Rev. Lett. 56, 2459 (1986).

[10] F. A. Berends and W. T. Giele, Recursive Calculations For Processes With N Gluons, Nucl. Phys. B306 (1988) 759. 
[11] F.A. Berends, R. Kleiss, P. De Causmaecker, R. Gastmans and T.T. Wu, Single Bremsstrahlung processes in gauge theories, Phys. Lett. B 103 (1981) 124;

P. De Causmaecker, R. Gastmans, W. Troost and T.T. Wu, Multiple Bremsstrahlung in gauge theories at high energies. 1. General formalism for quantum electrodynamics, Nucl. Phys. B 206 (1982) 53;

Z. Xu, D.-H. Zhang, L. Chang, Tsinghua University preprint TUTP-84/3 (1984), unpublished;

R. Kleiss and W.J. Stirling, Spinor techniques for calculating $p \bar{p} \rightarrow W^{ \pm} Z^{0}+$ jets, Nucl. Phys. B 262 (1985) 235;

J.F. Gunion and Z. Kunszt, Improved analytic techniques for tree graph

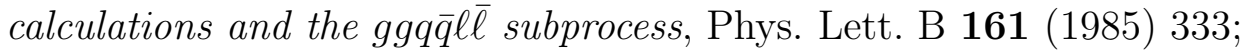

Z. Xu, D.-H. Zhang and L. Chang, Helicity amplitudes for multiple Bremsstrahlung in massless nonabelian gauge theories, Nucl. Phys. B 291, 392 (1987);

M.L. Mangano and S.J. Parke, Multiparton amplitudes in gauge theories, Phys. Rept. 200, 301 (1991).

[12] G. Georgiou and V.V. Khoze, Tree amplitudes in gauge theory as scalar MHV diagrams, JHEP 05 (2004) 070 hep-th/0404072.

[13] C.-J. Zhu, The googly amplitudes in gauge theory, JHEP 04 (2004) 032 [arXiv:hep-th/0403115].

[14] D.A. Kosower, Next-to-maximal helicity violating amplitudes in gauge theory, Phys. Rev. D71 (2005) 045007 [arXiv:hep-th/0406175].

[15] I. Bena, Z. Bern and D.A. Kosower, Twistor-space recursive formulation of gauge theory amplitudes, Phys. Rev. D71 (2005) 045008 hep-th/0406133.

[16] G. Georgiou, E.W.N. Glover and V.V. Khoze, Non-MHV tree amplitudes in gauge theory, JHEP 07 (2004) 048 hep-th/0407027.

[17] J.-B. Wu and C.-J. Zhu, MHV vertices and fermionic scattering amplitudes in gauge theory with quarks and gluinos, JHEP 09 (2004) 063 hep-th/0406146.

[18] L.J. Dixon, E.W.N. Glover and V.V. Khoze, MHV rules for Higgs plus multi-gluon amplitudes, JHEP 12 (2004) 015 hep-th/0411092.

[19] S.D. Badger, E.W.N. Glover and V.V. Khoze, MHV rules for Higgs plus multi-parton amplitudes, hep-th/0412275.

[20] Z. Bern, D. Forde, D.A. Kosower and P. Mastrolia, Twistor-inspired construction of electroweak vector boson currents, hep-ph/0412167. 
[21] Z. Bern, L.J. Dixon, D.C. Dunbar and D.A. Kosower, One loop n point gauge theory amplitudes, unitarity and collinear limits, Nucl. Phys. B425 (1994) 217-260 hep-ph/9403226.

[22] Z. Bern, L.J. Dixon, D.C. Dunbar and D.A. Kosower, Fusing gauge theory tree amplitudes into loop amplitudes, Nucl. Phys. B435 (1995) 59-101 hep-ph/9409265.

[23] F. Cachazo, P. Svrcek and E. Witten, Twistor space structure of one-loop amplitudes in gauge theory, JHEP 10 (2004) 074 hep-th/0406177.

[24] A. Brandhuber, B. Spence and G. Travaglini, One-loop gauge theory amplitudes in $N=4$ super yang-mills from $M H V$ vertices, Nucl. Phys. B706 (2005) 150 hep-th/0407214.

[25] F. Cachazo, Holomorphic anomaly of unitarity cuts and one-loop gauge theory amplitudes, hep-th/0410077.

[26] R. Britto, F. Cachazo and B. Feng, Computing one-loop amplitudes from the holomorphic anomaly of unitarity cuts, Phys. Rev. D71 (2005) 025012 hep-th/0410179.

[27] Z. Bern, V. Del Duca, L.J. Dixon and D.A. Kosower, All non-maximally-helicity-violating one-loop seven-gluon amplitudes in $N=4$ super-yang-mills theory, Phys. Rev. D71 (2005) 045006 hep-th/0410224.

[28] R. Britto, F. Cachazo and B. Feng, Generalized unitarity and one-loop amplitudes in $N=4$ super-yang-mills, hep-th/0412103.

[29] Z. Bern, L.J. Dixon and D.A. Kosower, All next-to-maximally-helicity-violating one-loop gluon amplitudes in $N=4$ super-yang-mills theory, hep-th/0412210.

[30] C. Quigley and M. Rozali, One-loop MHV amplitudes in supersymmetric gauge theories, JHEP 01 (2005) 053 hep-th/0410278.

[31] S.J. Bidder, N.E.J. Bjerrum-Bohr, L.J. Dixon and D.C. Dunbar, $N=1$ supersymmetric one-loop amplitudes and the holomorphic anomaly of unitarity cuts, Phys. Lett. B606 (2005) 189 hep-th/0410296.

[32] J. Bedford, A. Brandhuber, B. Spence and G. Travaglini, A twistor approach to one-loop amplitudes in $N=1$ supersymmetric yang-mills theory, hep-th/0410280.

[33] S.J. Bidder, N.E.J. Bjerrum-Bohr, D.C. Dunbar and W.B. Perkins, Twistor space structure of the box coefficients of $N=1$ one-loop amplitudes, hep-th/0412023. 
[34] R. Britto, E. Buchbinder, F. Cachazo and B. Feng, One-loop amplitudes of gluons in SQCD, arXiv:hep-ph/0503132.

[35] J. Bedford, A. Brandhuber, B. Spence and G. Travaglini, Non-supersymmetric loop amplitudes and MHV vertices, Nucl. Phys. B706 (2005) 100 hep-th/0412108.

[36] S.J. Bidder, N.E.J. Bjerrum-Bohr, D.C. Dunbar and W.B. Perkins, One-loop gluon scattering amplitudes in theories with $N<4$ supersymmetries, arXiv:hep-th/0502028.

[37] R. Roiban, M. Spradlin and A. Volovich, Dissolving $N=4$ loop amplitudes into QCD tree amplitudes, hep-th/0412265.

[38] R. Britto, F. Cachazo and B. Feng, New recursion relations for tree amplitudes of gluons, hep-th/0412308.

[39] R. Britto, F. Cachazo, B. Feng and E. Witten, Direct proof of tree-level recursion relation in Yang-Mills theory, hep-th/0501052.

[40] M.X. Luo and C.K. Wen, Recursion relations for tree amplitudes in super gauge theories, hep-th/0501121.

[41] M.X. Luo and C.K. Wen, Compact formulas for all tree amplitudes of six partons, hep-th/0502009.

[42] Z. Bern, L.J. Dixon and D.A. Kosower, On-shell recurrence relations for one-loop QCD amplitudes, hep-th/0501240.

[43] Z. Bern, L.J. Dixon and D.A. Kosower, The last of the finite loop amplitudes in $Q C D$, hep-ph/0505055.

[44] S.D. Badger, E.W.N. Glover, V.V. Khoze and P. Svrcek, Recursion relations for gauge theory amplitudes with massive particles, hep-th/0504159.

[45] F. Cachazo and P. Svrcek, Lectures on twistor strings and perturbative Yang-Mills theory, arXiv:hep-th/0504194.

[46] E. Witten, Perturbative gauge theory as a string theory in twistor space, Commun. Math. Phys. 252 (1004) 189 [arXiv:hep-th/0312171].

[47] D.A. Kosower, Multiple singular emission in gauge theories, Phys. Rev. D67 (2003) 116003 hep-ph/0212097.

[48] W. T. Giele and E.W.N. Glover, Higher order corrections to jet cross-sections in $e^{+} e^{-}$annihilation, Phys. Rev. D46 (1992) 1980-2010. 
[49] S. Frixione, Z. Kunszt, and A. Signer, Three-jet cross sections to next-to-leading order, Nucl. Phys. B467 (1996) 399-442, hep-ph/9512328.

[50] S. Catani and M.H. Seymour, A general algorithm for calculating jet cross sections in NLO QCD, Nucl. Phys. B485 (1997) 291-419, [ hep-ph/9605323.

[51] A. Gehrmann-De Ridder, T. Gehrmann and E.W.N. Glover, "Antenna Subtraction at NNLO," arXiv:hep-ph/0505111.

[52] Z. Bern and A. G. Morgan, Massive loop amplitudes from unitarity, Nucl. Phys. B467 (1996) 479-509, hep-ph/9511336.

[53] Z. Bern, L.J. Dixon, and D.A. Kosower, Progress in one-loop QCD computations, Ann. Rev. Nucl. Part. Sci. 46 (1996) 109 hep-ph/9602280.

[54] G. Altarelli and G. Parisi, Asymptotic freedom in parton language, Nucl. Phys. B126 (1977) 298.

[55] A. Bassetto, M. Ciafaloni, and G. Marchesini, Jet structure and infrared sensitive quantities in perturbative QCD, Phys. Rept. 100 (1983) 201

[56] Z. Bern and G. Chalmers, Factorization in one loop gauge theory, Nucl. Phys. B447 (1995) 465 hep-ph/9503236.

[57] Z. Bern, V. Del Duca, and C.R. Schmidt, The infrared behavior of one-loop gluon amplitudes at next-to-next-to-leading order, Phys. Lett. B445 (1998) 168 hep-ph/9810409.

[58] D.A. Kosower and P. Uwer, One-loop splitting amplitudes in gauge theory, Nucl. Phys. B563 (1999) 477 hep-ph/9903515.

[59] Z. Bern, V. Del Duca, W.B. Kilgore, and C.R. Schmidt, The infrared behavior of one-loop QCD amplitudes at next-to-next-to-leading order, Phys. Rev. D60 (1999) 116001, hep-ph/9903516.

[60] S. Catani and M. Grazzini, The soft-gluon current at one-loop order, Nucl. Phys. B591 (2000) 435 hep-ph/0007142.

[61] Z. Bern, L.J. Dixon, and D.A. Kosower, Two-loop $g \rightarrow$ g splitting amplitudes in QCD, JHEP 08 (2004) 012 hep-ph/0404293.

[62] S.D. Badger and E.W.N. Glover, Two-loop splitting functions in QCD, JHEP 07 (2004) 040 hep-ph/0405236.

[63] S. Catani, D. de Florian and G. Rodrigo, The triple collinear limit of one-loop amplitudes, Phys. Lett. B586 (2004) 323 hep-ph/0312067. 